\title{
Tendances de recherche dans le domaine des adhésifs à haute performance
}

\author{
G. RABILLOUD et B. SILLION
}

CEMOTA, BP. 3, 69390 Vernaison, France

\section{ABSTRACT}

The high performance adhesives must exhibit good mechanical properties in a wide range of temperatures in different environments for a long time.

The aromatic and heterocyclic structures are needed to obtain chemical and thermal resistance.

- The linear polymers offer good mechanical properties in term of fracture toughness, but they require high temperature and high pressure for the processing in order to insure good wettability. In addition, the temperature service is limited by the transition. Some examples of linear polyimide will be discussed.

- The crosslinkable systems are telechelic aromatic oligomers with end groups such as maleimides, acetylenes or nadimides. These systems offer good wettability, but the cure cycle is more complicated than the one of linear system. The mechanical properties are maintained up to $370^{\circ} \mathrm{C}$ for a short period of time.

- The blend of linear polymer and crosslinkable oligomers were studied in this case of epoxy resins and elastomers and thermoplastics.

Recently, it was mentioned that the blend of linear polyimides and crosslinkable bismaleimide or bis-nadimide improve the properties of each component, and these semi-IPN'S concept will be presented. 


\section{Introduction :}

Trois demandes fortes motivent les efforts de recherche et développement, toutes applications confondues dans le domaine des adhésifs : La résistance à la chaleur, la rapidité de mise en oeuvre, la non-toxicité (à la fois pour les manipulateurs et l'environnement).

Utilisés dans tous les domaines industriels, construction, mobilier, emballage, assemblages industriels automobiles, ils sont de plus en plus demandés dans les domaines de haute technologie automobile, biomédical, électronique, construction aéronautique.

D'une manière générale, l'assemblage par collage permet une jonction des pièces sur une plus grande surface et entraîne ainsi la disparition de points de contraintes au niveau des jonctions classiques.

Dans le domaine de l'automobile, c'est d'une part le développement des pièces en polymères qui provoque une augmentation du collage et d'autre part l'élévation de la température sous le capôt qui provoque une demande en adhésif plus résistants à la chaleur et aux huiles.

Dans l'industrie automobile, pratiquement toutes les familles chimiques : polyuréthane, phénoliques, acryliques, néoprènes, époxydes et époxydes modifiés, silicones, polyamide hot-melt, sont utilisés et les améliorations demandées sont plus satisfaites par des formulations que par le développement de nouveaux concepts.

Dans le domaine de l'aéronautique, la légèreté, les possibilités de collage de pièces composites sur des pièces métalliques, la transparence radar et la résistance à la chaleur sont les propriétés recherchées pour les adhésifs.

En ce qui concerne l'électronique, les adhésifs doivent présenter une grande pureté ionique, une bonne stabilité thermique. On recherche des prises rapides, des propriétés spécifiques du joint de colle pour éviter les contraintes mécaniques dues aux différences de coefficient d'expansion thermique du silicium et du substrat métallique.

D'autre part, on s'intéresse aux adhésifs conducteurs électriques et conducteurs thermiques.

A la fois pour les applications aérospatiales et électroniques, le matériau collé devra pouvoir travailler dans un large intervalle de température $\left(-50+300^{\circ} \mathrm{C}\right.$ pour l'aéronautique $)(-50$ $+150^{\circ} \mathrm{C}$ pour l'électronique).

Les époxydes et phénoliques sont limités aux applications à $180^{\circ} \mathrm{C}$ maximum.

La réflexion du chimiste doit donc s'orienter selon deux axes : obtenir des matériaux organiques résistant à la chaleur et présentant une bonne résilience. Or ces deux exigences sont assez contradictoires. En effet, les enchaînements aromatiques et hétérocycliques apportent la rigidité nécessaire pour l'obtention de transitions vitreuses ou cristallines élevées qui assurent la cohésion des chaînes à température élevée. En revanche, les structures travaillant à l'état vitreux présenteront une faible résilience. En première analyse, l'aptitude d'un polymère candidat à une application d'adhésion à haute température pourra s'apprécier par sa transition vitreuse, son énergie critique de fracture $\left(G_{1} C\right)$ et la détermination de la contrainte de rupture en cisaillement sur des éprouvettes de différentes natures.

Comme pour tous les systèmes polymères, il existe deux grandes familles de polymères aromatiques et hétérocycliques. Les systèmes linéaires et les oligomères réticulables (2). Du point de vue des applications adhésives, il est clair que les polymères doivent présenter une faible viscosité pour assurer le meilleur mouillage de la surface ; les polymères linéaires devront donc être mis en oeuvre à une température élevée supérieure à celle de leur 
transition vitreuse, mais en revanche, la structure linéaire leur assure une meilleure résilience $\left(G_{1} C\right.$ de 2 à $\left.4 \mathrm{KJ} / \mathrm{m} 2\right)$ que celle des systèmes réticulés $\left(G_{1} C\right.$ de 0,1 à $\left.0,3 \mathrm{KJ} / \mathrm{m} 2\right)$.

\section{Polymères hétérocycliques de haute masse (technique hot-melt)}

Depuis 1980, la NASA étudie les propriétés adhésives des polyimides amorphes ou semicristallins.

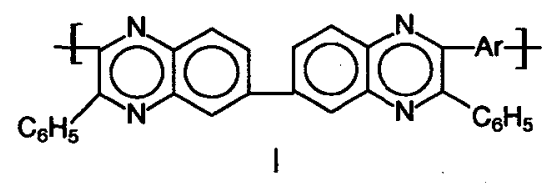<smiles></smiles><smiles>CC(C)(C)C1C(=O)c2ccc(Cl)cc2C1=O</smiles><smiles>Cc1ccc2c(c1)C(=O)N([Al]C(C)(C)C)C2=O</smiles><smiles>Cc1ccc(Oc2ccc(CC(C)[Al])cc2)cc1</smiles>

॥

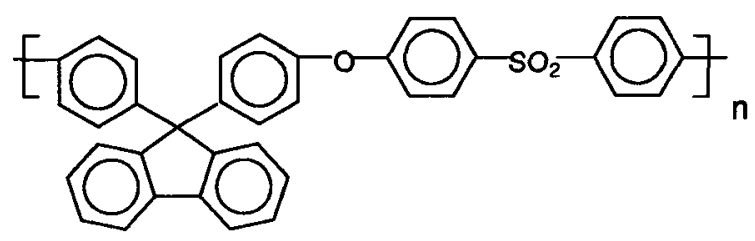

III

La comparaison d'une polyphénylquinoxaline I, d'un polyimide II et d'une polysulfone III de haute masse a été réalisée avec des éprouvettes de titane 6 Al4V et d'acier 17-7 PH (3).

Ces polymères doivent être mis en oeuvre à $371^{\circ} \mathrm{C}$ et $1,38 \mathrm{MPa}$ pour $1,343^{\circ} \mathrm{C}$ et $1,38 \mathrm{MPa}$ pour II et $343^{\circ} \mathrm{C}$ et $0,7 \mathrm{MPa}$ pour III. Les polymères I et II conservent leurs propriétés $20 \mathrm{MPa}$ après $3000 \mathrm{~h}$ à $232^{\circ} \mathrm{C}$. Le système III moins stable présente en outre l'inconvénient d'être sensible au Skydrol. II s'agit là de polymères étudiés sans charge d'aluminium.

\section{Mise en oeuvre de précurseurs de polymères linéaires}

L'objectif est de réaliser la polymérisation pendant la formation du joint de colle, c'est ce que l'on fait avec les époxydes et les polyuréthanes par exemple. Les polyimides peuvent être obtenus par la réaction suivante : 


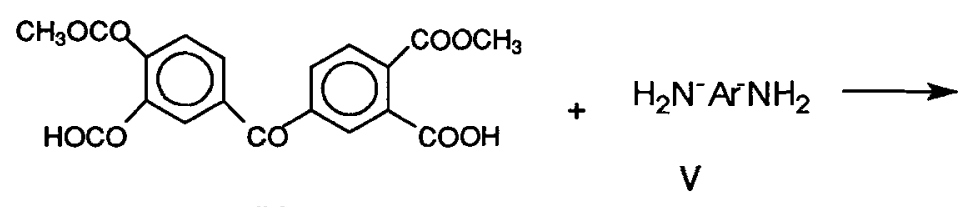

IV

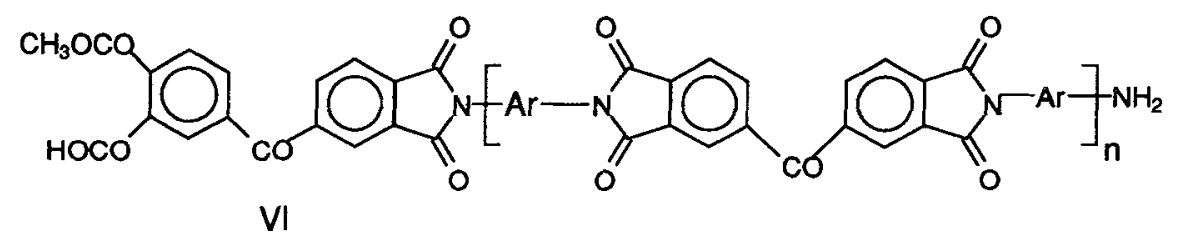

L'utilisation d'un mélange de diester IV et de diamine $\mathrm{V}$ conduit à un mélange fusible (déposable sur un tissu de verre) que l'on peut polymériser à $177^{\circ} \mathrm{C}$ sous faible pression 0,3 $\mathrm{MPa}$. Des collages sur titane présentent des valeurs de $14 \mathrm{MPa}$ à $260^{\circ} \mathrm{C}$ et de $8 \mathrm{MPa}$ à $260^{\circ} \mathrm{C}$ après $5000 \mathrm{~h}$.

Cette classe de produits, développée par American Cyanamid (FM 36), conduit à des résultats nettement supérieurs à ce que l'on peut obtenir avec des époxyphénoliques qui ne conservent leurs propriétés que $24 \mathrm{~h}$ à $260^{\circ} \mathrm{C}$ (4).

Dans le cas de ces structures polymères, la nature chimique joue un rôle déterminant en ce qui concerne la résistance à l'oxydation. Kohli a en effet (5) mis en évidence les propriétés exceptionnelles d'un polyimide fluoré VI, développé par Du Pont.

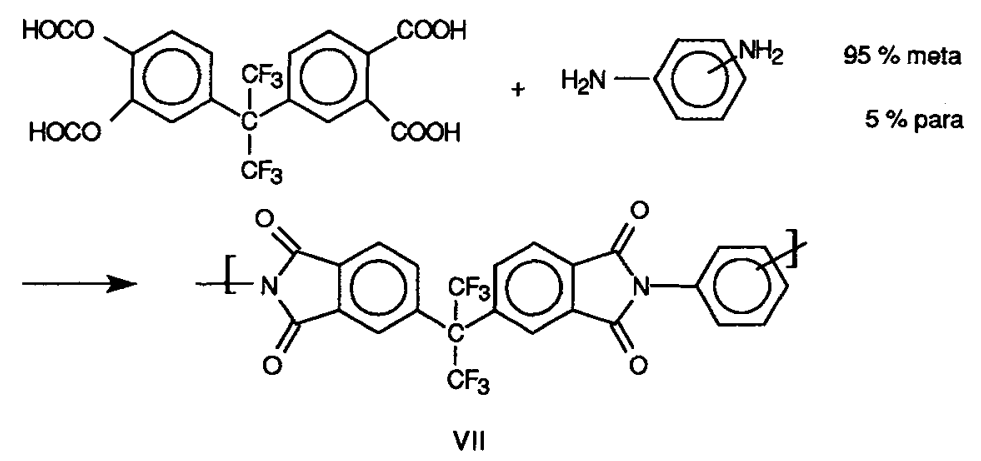

Le mélange, proposé par American Cyanamid peut être mis en oeuvre à $177^{\circ} \mathrm{C}$ et $0,3 \mathrm{MPa}$, suivi d'un post-traitement à $371^{\circ} \mathrm{C}$ sous pression.

Les collages sur titane présentent des propriétés de $16 \mathrm{MPa}$ après $5000 \mathrm{~h}$ à $260^{\circ} \mathrm{C}$ ou $9 \mathrm{MPa}$ après $1000 \mathrm{~h}$ à $315^{\circ} \mathrm{C}$ ou $0.7 \mathrm{MPa}$ après $125 \mathrm{~h}$ à $371^{\circ} \mathrm{C}$.

\section{Oligomères réticulables}

Ces composés sont des oligomères de masses moléculaires comprises entre 1000 et 2000 g.mol.-1 terminés à chaque extrémité par une liaison réactive. 


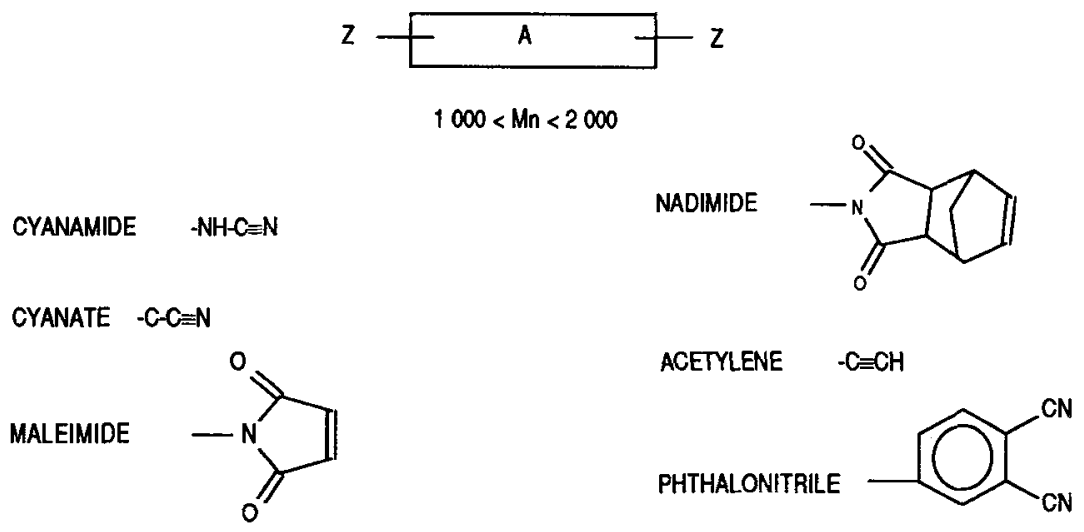

Les bismaléimides ont été commercialisés par Hysol en mélange avec des époxydes ; le produit EA 9673 est de cette famille; mis en oeuvre à $180^{\circ} \mathrm{C}$ avec un post-traitement à $250^{\circ} \mathrm{C}$, il conserve des propriétés pendant $150 \mathrm{~h}$ à $260^{\circ} \mathrm{C}(4)$.

Les bis-nadimides ont été commercialisés par American Cyanamid sous la marque FM 35. Leur cycle de mise en oeuvre commence par un chauffage à $204^{\circ} \mathrm{C}$ sous pression de 1,5 $\mathrm{MPa}$ et s'achève à $290^{\circ} \mathrm{C}$. Les propriétés d'éprouvettes de collage sur éprouvette en titane sont voisines de celles du polyimide de condensation FM 36, c'est à dire de l'ordre de $8 \mathrm{MPa}$ après $5000 \mathrm{~h}$ à $260^{\circ} \mathrm{C}$.

Les dérivés acétyléniques ont été commercialisés par National Starch. Les oligomères de type Thermid 600 ont été surtout proposés pour l'industrie électronique.

\section{Tendances de recherche}

\section{APPLICATIONS AEROSPATIALES}

II semble clair que les applications adhésives des polymères hétérocycliques de haute masse sont limités, d'une part par les difficultés de mise en oeuvre et par le comportement viscoélastique au-delà de $\mathrm{Tg}$. Le problème avec les polyimides de condensation est lié au fait que durant la mise en oeuvre, il se forme un dégagement de sous produits de la réaction (alcool et eau), ce qui est un handicap pour la réalisation de collages de grandes surfaces. Les oligomères qui réticulent par réaction d'addition ont l'inconvénient d'une très faible énergie critique de fracture préjudiciable au vieillissement sous contrainte.

Une orientation dont l'intérêt a été démontré dans le cas des matrices de composites consiste à explorer les mélanges de systèmes thermodurcissables et thermoplastiques.

Par mélange d'un polymère linéaire et d'oligomères de faible masse, on peut avoir une bonne miscibilité initiale de l'ensemble lorsque la polymérisation du système réticulable commence, la miscibilité diminue puisque dans l'équation définissant l'énergie libre d'un mélange de polymères, c'est le terme entropique qui est le plus important et il apparait une séparation de phase. Suivant la nature du mode de séparation, les phases peuvent adopter plusieurs types de morphologies : nodulaires, en éponge ou cocontinues. 


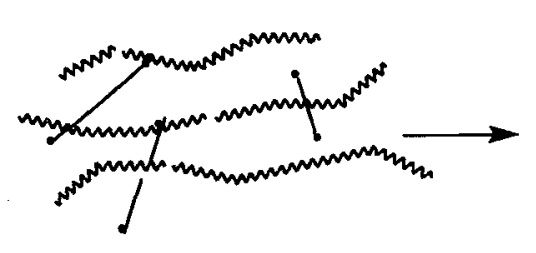

Mélange miscible

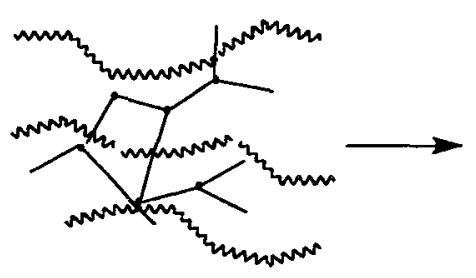

Début de polymérisation

Séparation de phase

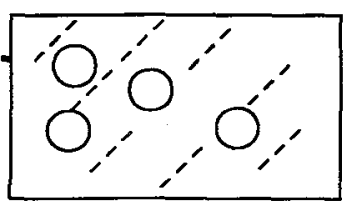

Séparation de

phase

L'amélioration des propriétés mécaniques : résistance à la propagation de fissure et énergie critique de fracture, est reliée à l'apparition de ces morphologies polyphasiques.

Dans le cas des époxydes, l'introduction d'une faible quantité (15 à $20 \%)$ de copolybutadiène acrylonitrile augmente l'énergie de fracture d'un facteur $30(6)$.

Cet effet a été expliqué par différents mécanismes : Formation de bandes de cisaillement (7), crazing (8), déchirure de la particule de caoutchouc (9) ou phénomène de cavitation au niveau du nodule de caoutchouc et déformation de la matrice initiée par le caoutchouc (10).

L'amélioration de la résilience apportée par l'élastomère qui a été mise à profit pour la formulation de colles pour l'électronique, se fait aux dépens de la stabilité thermique en raison de la diminution de la transition vitreuse.

Les réseaux époxydes tétrafonctionnels ont une transition vitreuse au voisinage de $200^{\circ} \mathrm{C}$ et l'on a examiné l'effet de mélanges avec des polysulfones aromatiques ou des polyétherimides. L'ensemble des résultats mettent en évidence l'influence de la séparation de phase et de la morphologie (11)(12) sur l'amélioration de la résilience sans porter préjudice à la stabilité thermique.

Dans le domaine des matériaux à hautes performances, les bismaléimides ont été modifiés par des polymères linéaires, par exemple le mélange de BMI et de polyimide ester permet de préparer des adhésifs pour la réalisation de circuits flexibles cuivre polyimide résistant à $300^{\circ} \mathrm{C}(13)$. Par ailleurs, avec une série de bismaléimides et de polyimides linéaires de structure chimique voisine, on obtient des mélanges partiellement miscibles dont les propriétés adhésives sont améliorées à température élevée (14). 
BAPPY : $X=N$; BAPB : $X=C H$<smiles>Nc1ccc(Cc2ccc(N)cc2)cc1</smiles>

MDA<smiles>[X]c1cccc(Oc2cccc(N3C(=O)CCC3=O)c2)c1</smiles>

PPY-BMI : $X=N$; PB-BMI : $X=\mathrm{CH}$<smiles>C[R16](C)(C)O</smiles><smiles>O=C1OC(=O)c2cc(Cl)ccc21</smiles>

BTDA

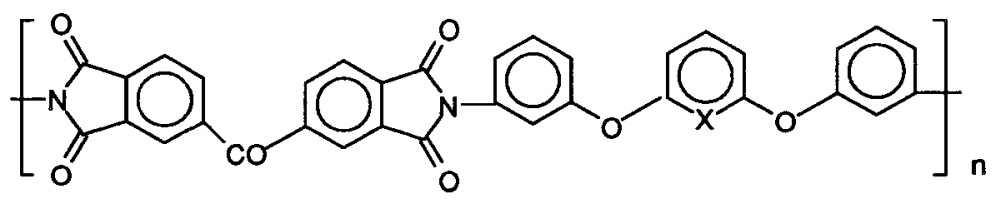

BAPPY-BTDA : $\mathrm{X}=\mathrm{N} ; \mathrm{BAPB-BTDA}: \mathrm{X}=\mathrm{CH}$

La comparaison entre des réseaux acétyléniques et bismaléimides de structure similaire, modifiés par un même polyimide (15) a été réalisée et l'on constate que les modules de conservation des réseaux sont dans l'ordre décroissant suivant :

Résine acétylénique polymère linéaire > bismaléimide polymère linéaire > polyimide linéaire seul

Ce qui indique une meilleure miscibilité pour le système acétylénique. En outre, les propriétés adhésives des réseaux semi-interpénétrés sont supérieures à celles du polymère linéaire à haute température. 
<smiles>C#Cc1ccc(C=Nc2cccc(Oc3cccc(Oc4cccc(N=Cc5ccc(C#C)cc5)c4)c3C=N)c2)cc1</smiles><smiles>[X]c1cccc(Oc2cccc(N3C(=O)CCC3=O)c2)c1</smiles>

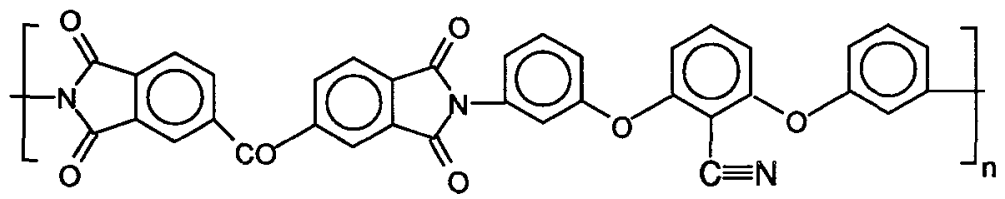

\section{APPLICATIONS MICROELECTRONIQUES}

Pour ce qui concerne les adhésifs à hautes performances, la chimie des polymères hétérocycliques apporte la solution au problème de la stabilité thermique ; de plus, les nouveaux concepts de mélange de polymères permettent d'améliorer les propriétés dans un large intervalle de température et de répondre aux applications spécifiques d'industries de la microélectronique.

Les industries électroniques et aérospatiales ont une exigence commune qui est la fiabilité à long terme et dans des conditions d'environnement sévères des ensembles ou des sousensembres constituant un système fonctionnel. Cela implique pourquoi l'emploi des adhésifs ne s'est généralisé que progressivement depuis 1970 sous l'impulsion des travaux de la NASA dans le cadre du programme Apollo. De façon simplifiée, un ensemble électronique est constitué d'un substrat d'interconnexion rigide ou flexible sur lequel sont reportés des éléments actifs, les circuits intégrés, et des éléments passifs : résistances, condensateurs, lignes à retard, etc. Tous ces composants sont soudés sur les plots de contact de la carte au cours de l'opération finale de montage. L'utilisation d'adhésifs électroconducteurs pour remplacer la soudure étain-plomb reste encore marginale dans les fabrications de grands volumes.

Par contre, les adhésifs conducteurs ont connu un développement considérable dans l'industrie des semiconducteurs avec la généralisation de la technologie d'encapsulation des circuits intégrés dans des boitiers plastiques. A la fin du cycle de fabrication sur une tranche de silicium, les circuits intégrés, dont la taille varie de $2 \mathrm{~mm} 2$ à plus d'1 $\mathrm{cm} 2$ sont découpés et fixés sur un peigne métallique dont les pattes ont pour rôle d'assurer les interconnexions électriques avec le monde extérieur. Le système formé par le circuit intégré et le peigne est ensuite enfermé dans un boitier protecteur en céramique, en métal ou en plastique. Les adhésifs électroconducteurs ne peuvent être utilisés qu'avec les boitiers plastiques dont la température de polymérisation est inférieure à $200^{\circ} \mathrm{C}$. C'est un marché considérable car il couvre $90 \%$ de la production de circuits intégrés, soit plusieurs milliards de pièces par an. 
Le collage du circuit intégré sur une embase métallique assure trois fonctions : mécanique, électrique et thermique. La première est commune à tous les adhésifs structuraux en ce sens que les propriétés mécaniques de l'assemblage dépendent principalement du polymère utilisé pour préparer l'adhésif. La conductivité électrique est apportée par l'addition d'une charge métallique, généralement faite d'un mélange de paillettes et de poudre d'argent. Une résistivité volumique de 0,1 à $1 \mathrm{~m} \Omega$. suffit pour éliminer les charges statiques et mettre le semiconducteur au potentiel de masse. La charge métallique permet également un transfert de la chaleur émise par le circuit vers l'extérieur par l'intermédiaire du peigne métallique.

Dans le cas des semiconducteurs de puissance, le film adhésif doit avant tout servir de conducteur thermique car la température de la jonction peut atteindre facilement $200^{\circ} \mathrm{C}$. Les compositions adhésives sont préparées avec des polymères thermostables comme les polyimides alliés à des charges minérales thermoconductrices : oxyde de béryllium, nitrure d'aluminium ou poudre de diamant. La conductivité thermique, qui est de $0,1 \mathrm{~W} / \mathrm{m}$. K pour une résine époxyde, atteint $3 \mathrm{~W} / \mathrm{m} . \mathrm{K}$ lorsque la résine est chargée avec du nitrure d'aluminium et $10 \mathrm{~W} / \mathrm{m} . \mathrm{K}$ quand elle contient du diamant.

Les adhésifs utilisés en électronique pour le collage des circuits intégrés sont formulés à partir de résines époxydes ( $90 \%$ de la production) ou de polyimides pour les composants qui nécessitent une oplus grande résistance à la chaleur. Dans chacune de ces familles, le choix des constituants organiques : résines, durcisseurs, catalyseurs, solvants, etc. est dicté par les conditions de mise en oeuvre d'une part et par les propriétés requises au niveau de l'assemblage final.

Les adhésifs époxydes dits de première génération sont pour la plupart des systèmes bicomposants dont le mélange est effectué par l'utilisateur. Une formulation type proposée par plusieurs producteurs est composée d'une première partie contenant le diglycidyl éther du bis-phénol F, une époxy-novolaque et $70 \%$ de paillettes d'argent. La seconde partie est un mélange de $\gamma$-butyrolactone, de 2-éthyl-4-méthyl-1-(2-cyanoéthyl)-imidazole et de paillettes d'argent. La cuisson du mélange est effectuée à $120-140^{\circ} \mathrm{C}$ pendant 30 minutes pour donner un adhésif ayant une température de transition vitreuse de $95^{\circ} \mathrm{C}$ et une résistance au cisaillement de $10 \mathrm{MPa}$.

Le dicyandiamide combiné à une urée a été longtemps utilisé comme système catalytique latent dans les formulations monocomposant, à base de diglycydyl éther du bisphénol-A, de monoépoxyde (diluants réactifs) et de charges métalliques. Les adhésifs contenant du dicyandiamide présentent cependant l'inconvénient de dégager de l'ammoniac pendant les tests de vieillissement en chaleur humide.

Les temps de cuisson requis par les adhésifs époxydes ne permettent pas d'inclure l'étape de collage dans une ligne de production continue puisque les éléments à coller doivent être introduits et laissés dans l'étuve de cuisson pendant 1 à 2 heures. La solution à ce problème est apportée par de nouveaux adhésifs à prise rapide dont la polymérisation s'effectue en une vingtaine de secondes à $150^{\circ} \mathrm{C}$. Cette opération peut être réalisée sur une plaque chauffante de longueur adéquate sur laquelle circulent les pièces à coller. Ces adhésifs contiennent un mélange de résine époxyde, d'époxynovolaque et un système de catalyseurs très actifs à base de divers imidazoles. A la température ambiante, la durée de vie en pot ne dépasse pas quelquelques heures de sorte que l'adhésif doit être stocké et transporté à $40^{\circ} \mathrm{C}$. Les adhésifs à prise rapide connaîtront un développement considérable lorsque des catalyseurs peu actifs à $20^{\circ} \mathrm{C}$ et hautement réactifs au-dessus de $120^{\circ} \mathrm{C}$ auront été mis au point.

Les circuits intégrés modernes atteingent couramment une surface supérieure à $1 \mathrm{~cm} 2 \mathrm{de}$ sorte que les contraintes mécaniques qui se développent pendant la cuisson de l'adhésif 
peuvent initier des zones de fracture et détruire le curcuit. Un calcul simple pour estimer le niveau des contraintes thermiques peut être fait en utilisant l'équation à une dimension :

$$
\sigma=\left(\alpha_{\mathrm{ad}}-\alpha_{\mathrm{si}}\right) \Delta \mathrm{T} \times \mathrm{E}_{\mathrm{ad}}
$$

ou $\sigma$ représente la contrainte (tension ou compression), $\alpha$ ad et $\alpha$ si sont les coefficients d'expansion thermique de l'adhésif et du silicium, $\Delta T$ est la différence entre la température de transition vitreuse et la température de test est $E_{\mathrm{ad}}$ est le module d'Young de l'adhésif.

Le coefficient d'expansion thermique des adhésifs époxydes (45 ppm/ $\left.{ }^{\circ} \mathrm{C}\right)$ est beaucoup plus élevé que celui du silicium $\left(3 \mathrm{ppm} /{ }^{\circ} \mathrm{C}\right)$ et il ne peut être réduit de façon sensible. Une valeur de $150^{\circ} \mathrm{C}$ pour la température de transition vitreuse est considérée comme un bon compromis pour supporter les tests de vieillissement en chaleur humide. Dans ces conditions, un adhésif conventionnel ayant un module de $9,8 \mathrm{GPa}$ peut induire des contraintes mécaniques supérieures à $300 \mathrm{MPa}$. L'une des méthodes employěes pour réduire le niveau des contraintes est de faire appel à des adhésifs plus flexibles ayant un module d'élasticité beaucoup plus bas. Même si le coefficient d'expansion thermique est plus élevé (120 $\mathrm{ppm} /{ }^{\circ} \mathrm{C}$ ), un adhésif ayant un module de $0,14 \mathrm{GPa}$ génère des contraintes mécaniques de l'ordre de 2 à $5 \mathrm{MPa}$ bien inférieures au seuil de rupture du silicium.

Les adhésifs à relaxation de contraintes ayant une transition vitreuse proche de $150^{\circ} \mathrm{C}$ sont en général préparés à partir d'un mélange de résines époxydes, d'un durcisseur et d'un polymère linéaire largement incompatible avec la matrice époxyde. Les polymères les plus employés pour cette application sont les copolymères acrylonitrile-butadiène terminés par des fonctions acide carboxylique réactives vis-à-vis des résines époxydes. Une composition type contient par exemple $70 \%$ de résine époxyde, $30 \%$ de caoutchouc nitrile (CTBN-1300), un imidazole et la charge de paillettes d'argent nécessaire pour assurer la conductivité électrique.

Les recherches effectuées dans le domaine des adhésifs à faibles contraintes portent pratiquement toutes sur des mélanges de polymères incompatibles. Les époxydes restent le constituant de base de la matrice thermodurcissable pour leurs excellentes propriétés adhésives et les possibilités d'en modifier la rhéologie. En ce qui concerne le second polymère, les polydiméthylsilicones et les polyméthylphénylsilicones présentent sur les caoutchoucs nitriles ou les polybutadiènes l'avantage d'une meilleure stabilité thermique.

La seconde famille d'adhésifs conducteurs, celle des polyimides, n'est employée que dans la fabrication des composants à haute fiabilité : matériel militaire, télécommunications et systèmes professionnels. Seuls les prépolymères de condensation ont connu un succès commercial malgré les problèmes liés à la température de cuisson élevée $\left(280-300^{\circ} \mathrm{C}\right)$ et à l'évolution de composés volatils pendant la polymérisation. Les adhésifs thermoconducteurs à base de polyimides et de nitrure d'aluminium ou de diamant devraient connaître une rapide expansion dans le domaine des semiconducteurs de puissance. Ce sont actuellement les seuls adhésifs capables de travailler au-dessus de $250^{\circ} \mathrm{C}$ en service continu tout en conservant de très bonnes propriétés adhésives.

\section{REFERENCES}

(1) REISCH M.S., Chemical and Engineering News, March 4, (1991), 23

(2) SILLION B., Polyimides and Other Heteroaromatic Polymers, Chapt. 30 (pp 499-532), Vol. 5 "Step Polymerization" - COMPREHENSIVE POLYMER SCIENCE, Pergamon Press, 1 st Ed. 1989 
(3) HENDRICKS C.L., HILL S.G., HALE J.N., DUMARS W.G., NASA Contractor Report 178176, 1987

(4) CLER S. , Club Français du SAMPE, Toulouse 1991

(5) KOHLI D./K., 37th International SAMPE Symposium, March 9-12 (1992), 430

(6) BASCOM W. D., COTTINGTON R.L., JONES R.C., PEYSER P., J. Appl. Polym. Sci., 19, (1975), 2545

(7) Mc GARRY F.S., Proc. Roy. Soc. London, A319, (1970) 59

(8) CHAN L.C., GILHAM J.K. , KINLOCH A.J., SHAWS J., In Adv. Chemistry Series 209, ACS 1984

(9) KUNZ S. et BEAUMONT P.W.R. ; J. Mat. Sci. 16, (1981), 3141

(10) KINLOCH A.J., SHOW S.J., TODD D.A., Polymer , 24, (1983), 1341

(11) SEFTON M.S., MC GRAIL P.T., PEACOK J.A., WILKINSON S.P., CRICK P.A., DAVIES M., ALMEN G. ; 19th International SAMPE Technical Conf., October 13-15, (1987), 700

(12) ALMEN G.R., MACKENZIE P., MALHOTRA V., MASKELL R.K., MC GRAIL P.T., SEFTON M.S., 20th International SAMPE Symposium, March 7-10, (1988), 970

(13) TURPIN R.L., GREEN A.,35th International SAMPE Symposium, March 7-10, (1988)

(14) PASCAL T., MERCIER R., SILLION B., Polymer, 31, (1990), 83

(15) PASCAL T., SILLION B., GROSJEAN F., GRENIER-LOUSTALOT M.F., GRENIER P., High Performance Polymer, 2, (1990), 95 